\title{
Chemotherapy for Liver Metastasis Originating from Colorectal Cancer with Portal Vein Tumor Thrombosis: A Case Report
}

\author{
Atsushi Kawasaki $^{a} \quad K^{2}$ ji Mimatsu ${ }^{a}$ Takatsugu Oida $^{a}$ Hisao Kano $^{a}$ \\ Youichi Kuboi $^{a}$ Nobutada Fukino $^{a}$ Kazutoshi Kida $^{a}$ Sadao Amano ${ }^{b}$ \\ ${ }^{a}$ Department of Surgery, Social Insurance Yokohama Central Hospital, Yokohama, and \\ ${ }^{b}$ Department of Surgery, Nihon University School of Medicine, Tokyo, Japan
}

\section{Key Words}

Colorectal cancer $\cdot$ Portal vein tumor thrombosis · Panitumumab

\begin{abstract}
The patient was a male in his 70s with a history of chronic renal failure and dilated cardiomyopathy. In January 2011, he underwent abdominoperineal resection of the rectum, right hepatic lobectomy, and resection of a portal vein tumor thrombus with a diagnosis of rectal cancer and metastatic liver cancer accompanied by portal vein tumor thrombosis. Although 5-fluorouracil + I-leucovorin therapy (RPMI regimen) was carried out as postoperative adjuvant chemotherapy, the tumor marker (CEA and VA19-9) levels increased 8 months after surgery. Since the functions of major organs were impaired, UFT ${ }^{\circledR}+\mathrm{UZEL}^{\circledR}$ therapy was started. The tumor marker levels decreased temporarily, but increased again 12 months after surgery, and so intravenous instillation of panitumumab was initiated. Nine administrations have been performed to date, with no increase in tumor marker levels or exacerbation of the condition. Also, no grade 2 or severer adverse event has been noted according to CTCAE v.4.0. The experience with this patient suggests the possibility that exacerbation of the condition of patients with liver metastasis of colorectal cancer accompanied by portal vein tumor thrombosis with abnormalities in the functions of major organs can be controlled temporarily by the administration of panitumumab alone.
\end{abstract}




\section{Introduction}

The prognosis of liver metastasis of colorectal cancer accompanied by portal vein tumor thrombosis has been reported to be relatively favorable after appropriate hepatectomy and removal of the tumor thrombus [1], but the possibility of the presence of residual tumor cells is high, and postoperative adjuvant chemotherapy is strongly recommended as much as possible. In this report, we present postoperative chemotherapy and chemotherapy for recurrence that we performed in a case of liver metastasis of colorectal cancer accompanied by portal vein tumor thrombosis, along with a review of the literature.

\section{Case Report}

The patient was a male in his 70s who consulted a local physician with malaise as the primary complaint. He was referred to our hospital because a tumoral lesion was detected in the right hepatic lobe by ultrasonography. The patient had been medicated for chronic renal failure and dilated cardiomyopathy. Abdominal contrast-enhanced CT performed at our hospital confirmed a tumoral lesion of $90 \times 70 \mathrm{~mm}$ in the right hepatic lobe. Tumor thrombosis was also noted in the main trunk of the portal vein (fig. 1a). Lower digestive tract radiography performed to search the primary lesion revealed circumferential stenosis due to a mass of $50 \mathrm{~mm}$ in diameter from the upper (Ra) to lower (Rb) rectum. Abdominal angiography showed tumor thrombosis from the right branch to the main trunk of the portal vein. Regarding tumor markers, the CEA (carcinoembryonic antigen) and CA19-9 (carbohydrate antigen) levels were markedly elevated to $596.4 \mathrm{ng} / \mathrm{ml}$ (normal range $<5 \mathrm{ng} / \mathrm{ml}$ ) and $153 \mathrm{U} / \mathrm{ml}$ (normal range $<37 \mathrm{U} / \mathrm{ml}$ ), respectively. From these results, a diagnosis of liver metastasis of rectal cancer accompanied by tumor thrombosis of the portal vein was made, and open abdominal surgery was performed in January 2011. The surgery consisted of abdominoperineal rectal resection (with D3 lymph node dissection), right hepatic lobectomy, and resection of the portal vein tumor thrombus. The postoperative course was uneventful, and the patient was discharged on the 14th postoperative day. The histopathological diagnosis was moderately differentiated adenocarcinoma, and the final staging according to the TMN classification was stage IV (T3, N0, HEP).

As postoperative adjuvant chemotherapy, 3 courses of 5 -fluorouracil $\left(600 \mathrm{mg} / \mathrm{m}^{2} /\right.$ day $)$ + l-leucovorin $\left(250 \mathrm{mg} / \mathrm{m}^{2} /\right.$ day) therapy (RPMI regimen) were carried out (total number of administrations, 18). In May 2011 (4 months after surgery), the tumor marker levels were normalized, and no recurrence or metastasis was noted by imaging examinations at the end of the adjuvant chemotherapy. However, the tumor marker levels increased again (CEA: 14.2 $\mathrm{ng} / \mathrm{ml}, \mathrm{CA} 19-9: 34 \mathrm{U} / \mathrm{ml}$ ) in September 2011 (8 months after surgery), and tumoral lesions suggestive of recurrence were noted in the lateral segment of the liver and left lung by CT performed 9 months after surgery (fig. 1b). A diagnosis of unresectable recurrence of colorectal cancer was made, and the introduction of FOLFOX6 or FOLFIRI therapy was evaluated but abandoned as the cardiac and renal functions were reduced with a left ventricular ejection fraction measured by echocardiography of $41.1 \%$ and serum creatinine levels of $2.5-3.2 \mathrm{mg} / \mathrm{dl}$ (normal range $0.66-1.13 \mathrm{mg} / \mathrm{dl}$ ), and oral uracil/tegafur (UFT ${ }^{\circledR}$ ) + folinate (UZEL ${ }^{\circledR}$ ) therapy ( $500 \mathrm{mg} /$ day UFT $+75 \mathrm{mg} /$ day UZEL, 3-week administration with 1 -week rest) was initiated. While the tumor marker levels were temporarily reduced after the beginning of UFT + UZEL therapy, they increased again in January 2012 (12 months after surgery). Since the KRAS gene was the wild type, and EGFR (epidermal growth factor receptor) was positive, intravenous instillation of panitumumab (Vectibix ${ }^{\circledR}$ ) was initiated (6 
Kawasaki et al.: Chemotherapy for Liver Metastasis Originating from Colorectal Cancer with Portal Vein Tumor Thrombosis: A Case Report

$\mathrm{mg} / \mathrm{kg}$ once every 2 weeks). The tumor marker levels were markedly reduced after the intravenous instillation of panitumumab 2 times. Although grade 1 skin disorder (acne-like rash on the face and head) according to the Common Terminology Criteria for Adverse Events v.4.0 appeared, no severe adverse event such as infusion reaction and electrolyte abnormality was noted. The intravenous instillation of panitumumab has been performed 9 times to date, and no increase in the tumor marker level has been observed (fig. 2).

\section{Discussion}

The frequency of detecting portal vein tumor thrombosis in patients with metastatic liver cancer is relatively low, and Albacete et al. [2] reported this frequency as 5\% according to a review of autopsy findings. Also, gross portal vein tumor thrombosis was reported to be confirmed in 4 (2.8\%) of 142 patients with metastatic liver cancer from colorectal cancer [3].

Surgery is the first choice for liver metastasis of colorectal cancer [4], but only 15-35\% of patients have indications for hepatectomy [5, 6]. For those who have no indication for surgical resection, oxaliplatin + infusional fluorouracil + l-leucovorin (FOLFOX) therapy, irinotecan + infusional fluorouracil + l-leucovorin (FOLFIRI) therapy, and various molecularly targeted drugs have been recently introduced with improvements in therapeutic outcomes.

The prognosis of patients with liver metastasis of colorectal cancer accompanied by portal vein tumor thrombosis has been reported to be relatively favorable after resection of the areas of the liver supplied by the portal system and removal of the tumor thrombus [1]. However, as the possibility of the presence of residual tumor cells is high, postoperative adjuvant chemotherapy should be performed as much as possible. In our patient, also, 5fluorouracil + l-leucovorin therapy was carried out as postoperative adjuvant chemotherapy, but recurrence and metastasis occurred in the liver and lung 9 months after surgery. There have been few reports describing postoperative adjuvant chemotherapy for liver metastasis of colorectal cancer accompanied by portal vein tumor thrombosis, and, to our knowledge, only 2 cases treated by UFT + UZEL therapy have been reported since 2000 [7, 8]. Many patients with liver metastasis of colorectal cancer accompanied by portal vein tumor thrombosis were reported to have developed metastasis or recurrence about 1 year after surgery and died, and some postoperative adjuvant chemotherapy is considered to have been conducted in many patients. In case reports in which postoperative adjuvant chemotherapy was not performed, refusal of the patients was mentioned as its reason [9]. Our patient showed recurrence and metastasis in the liver and lung after the end of postoperative adjuvant chemotherapy. Although we initially evaluated FOLFOX therapy or capecitabine + oxaliplatin (CapOX) therapy as postoperative adjuvant chemotherapy, we abandoned it because of concern over exacerbation of the renal function. In addition, the regimens of chemotherapy that could be introduced after recurrence were restricted by the possibility of exacerbation of the renal and cardiac function.

Panitumumab is a fully human IgG2 anti-EGFR monoclonal antibody drug and is considered to be comparable in usefulness to cetuximab (Erbitux ${ }^{\circledR}$ ), another anti-EGFR antibody drug. However, panitumumab has the advantages that it may be administered once every 2 weeks and that, being a fully human antibody, it less frequently causes infusion reactions. It can also be used alone over a relatively long period even in and after the tertiary treatment with attention to adverse events such as skin disorders (acne-like rash, perionychium inflammation), diarrhea, and hypomagnesium. In our patient, we performed the intravenous instillation of panitumumab alone 9 times, and a state of stable disease could be maintained 
for about 3 months. The experience with this patient suggests the possibility that the exacerbation of the condition may be controlled for a period in patients with liver metastasis of colorectal cancer accompanied by portal vein tumor thrombosis with abnormalities in major organ functions by the administration of panitumumab alone.

\section{References}

1 Tomimaru Y, Sasaki Y, Yamada T, Gotoh K, Noura S, Eguchi H, Miyashiro I, Ohue M, Ohigashi H, Yano M, Ishikawa $\mathrm{O}$, Imaaka S: Liver metastasis originating from colorectal cancer with macroscopic portal vein tumor thrombosis: a case report and review of the literature. J Med Case Rep 2010;4:382.

-2 Albacete RA, Matthews MJ, Saini N: Portal vein thromboses in malignant hepatoma. Ann Intern Med 1967;67:337-348.

-3 Tada K, Kokudo N, Seki M, Ueno M, Azekura K, Ohta H, Yamaguchi T, Matsubara T, Takahashi T, Nakajima T, Yanagisawa A, Muto T: Hepatic resection for colorectal metastasis with macroscopic tumor thrombus in the portal vein. World J Surg 2003;27:299-303.

4 Minagawa M, Makuuchi M, Torzilli G, Takayama T, Kawasaki S, Kosuge T, Yamamoto J, Imamura H: Extension of the frontiers of surgical indication in the treatment of liver metastases from colorectal cancer: long-term results. Ann Surg 2000;231:487-499.

-5 Adam R, Pascal G, Azoulay D, Tanaka K, Castaing D, Bismuth H: Liver resection for colorectal metastases: the third hepatectomy. Ann Surg 2003;238:871-883.

6 Petrelli NJ, Abbruzzese J, Mansfield P, Minsky B: Hepatic resection: the last surgical frontier for colorectal cancer. J Clin Oncol 2005;23:4475-4477.

-7 Sugiura T, Nagino M, Ebata T, Arai T, Oda K, Yuasa N, Nimura Y: Treatment of colorectal liver metastasis with biliary and portal vein tumor thrombi by hepatopancreatoduodenectomy. J Hepatobiliary Pancreat Surg 2006;13:256-259.

8 Inokawa Y, Kajikawa M, Tsushima Y, Takase T, Nakayama S, Yaguchi T: A case of portal vein tumour thrombosis following rectal cancer surgery unaccompanied by hepatic parenchyma metastasis. J Jpa Surg Assoc 2009;70:824-828 (in Japanese with English abstract).

-9 Oikawa T, Takayama T, Okada S, Kamo T, Sugitani M, Sakamoto M: Macroscopic portal vein tumor thrombi of liver metastasis from colorectal cancer. J Hepatobiliary Pancreat Surg 2009;16:90-93. 


\section{Case Reports in Oncology}

\begin{tabular}{l|l}
\hline Case Rep Oncol 2013;6:275-279 \\
\hline DOI: 10.1159/000343680 & $\begin{array}{l}\text { C 2013 S. Karger AG, Basel } \\
\text { www.karger.com/cro }\end{array}$ \\
\hline
\end{tabular}

Kawasaki et al.: Chemotherapy for Liver Metastasis Originating from Colorectal Cancer with Portal Vein Tumor Thrombosis: A Case Report
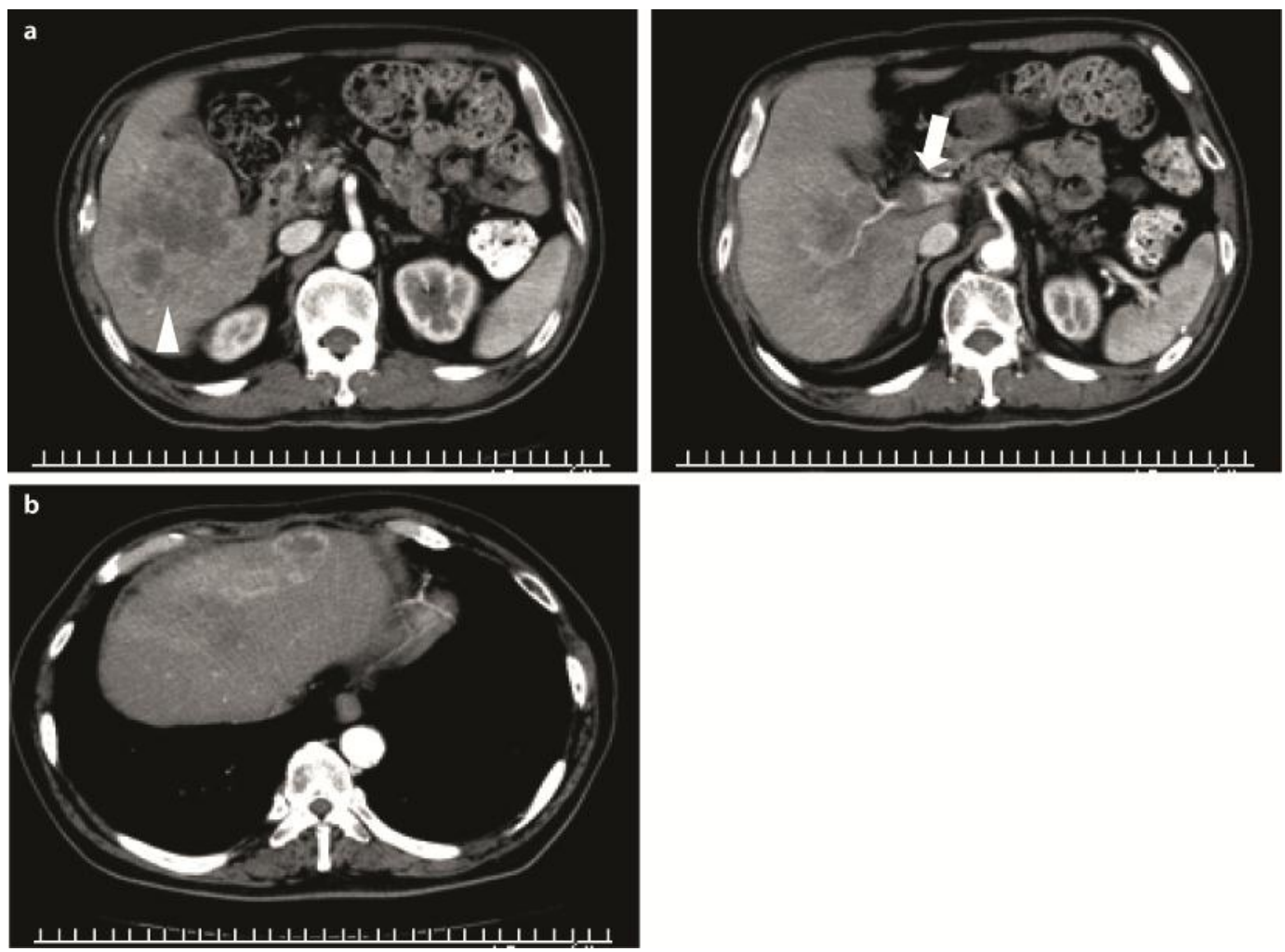

Fig. 1. Abdominal contrast-enhanced CT. a At initial examination. Abdominal contrast-enhanced CT demonstrated a large tumor about $90 \times 70 \mathrm{~mm}$ in the right hepatic lobe (arrowhead), and a tumor thrombus in the portal vein (arrow). b Nine months after surgery. Tumoral lesions suggestive of recurrence were noted in the lateral segment of the liver.

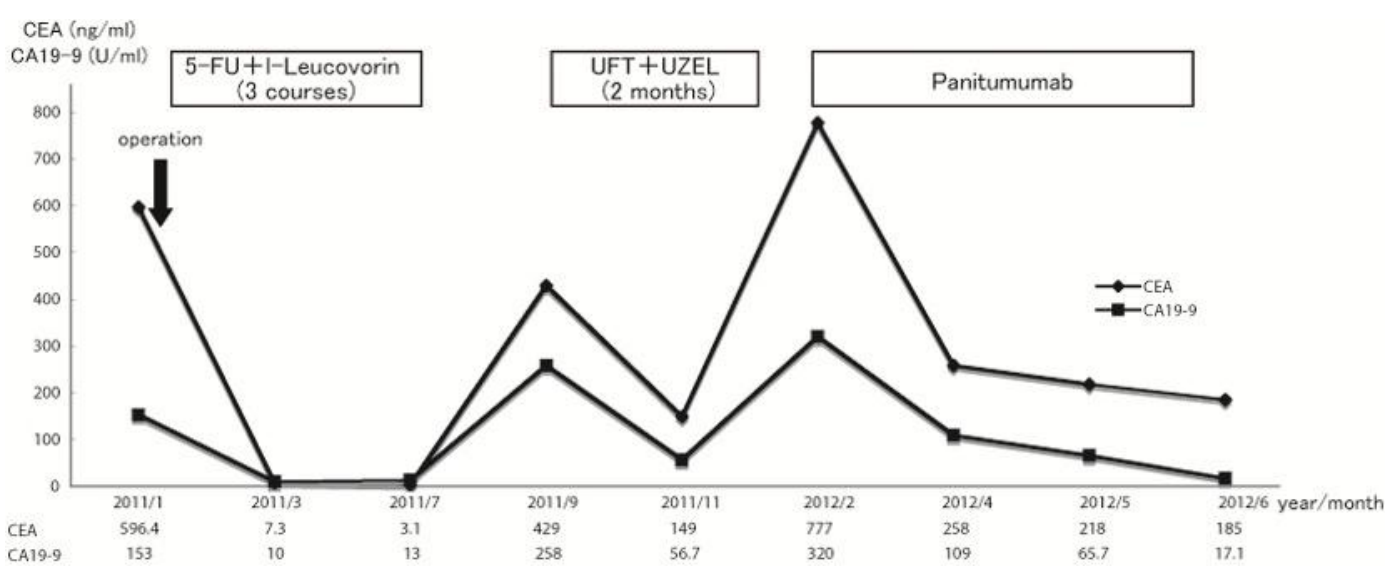

Fig. 2. Treatment course and changes in tumor markers. 2011/1: before surgery. 2011/3: after surgery. 2011/7: after 3 courses of 5-fluorouracil (5-FU) + l-leucovorin therapy. 2011/9: before initiation of UFT + UZEL therapy. 2012/2: before initiation of panitumumab therapy. 2012/6: after 9 times of panitumumab therapy. 